\title{
Effects of morphology on the micro-compression response of carbon nanotube forests ${ }^{\dagger}$
}

\author{
Parisa Pour Shahid Saeed Abadi, ${ }^{a}$ Shelby B. Hutchens, ${ }^{b}$ Julia R. Greer, ${ }^{b}$ Baratunde A. Cola ${ }^{* a c}$ \\ and Samuel Graham*ac
}

\author{
Received 27th February 2012, Accepted 27th March 2012 \\ DOI: 10.1039/c2nr30474k
}

\begin{abstract}
This study reports the mechanical response of distinct carbon nanotube (CNT) morphologies as revealed by flat punch in situ nanoindentation in a scanning electron microscope. We find that the location of incipient deformation varies significantly by changing the CNT growth parameters. The initial buckles formed close to the growth substrate in 70 and $190 \mu \mathrm{m}$ tall CNT forests grown with low pressure chemical vapor deposition (LPCVD) and moved to $\sim 100 \mu \mathrm{m}$ above the growth substrate when the height increased to $280 \mu \mathrm{m}$. Change of the recipe from LPCVD to CVD at pressures near atmospheric changed the location of the initial buckling event from the bottom half to the top half of the CNT forest. Plasma pretreatment of the catalyst also resulted in a unique CNT forest morphology in which deformation started by bending and buckling of the CNT tips. We find that the vertical gradients in CNT morphology dictate the location of incipient buckling. These new insights are critical in the design of CNT forests for a variety of applications where mechanical contact is important.
\end{abstract}

\section{A Introduction}

Carbon nanotube (CNT) forests possess unique properties (e.g., high thermal and electrical conductivity, optical absorption, and mechanical compliance) which have rendered them as excellent candidates for applications such as field emitters, ${ }^{1}$ thermal ${ }^{2,3}$ and electrical ${ }^{4}$ interfaces, super dark absorbers, ${ }^{5,6}$ and through wafer interconnects. ${ }^{7}$ The collective mechanical response of $\mathrm{CNT}$ forests to compression loading has recently been the focus of many studies ${ }^{8-33}$ because this response determines their performance when they are in contact with other materials during either device fabrication or in-use. Though the structure of CNT forests appears to be vertically aligned on the scale of tens or hundreds of microns, at higher magnifications the individual CNTs are clearly discernible and exhibit various degrees of misalignment and entanglement. The differences in the density and tortuosity of the CNTs are expected to lead to vertical variations in interaction among the tubes within the forests, which governs their mechanical response under loading. Thus, their complex and intertwined morphology must be investigated

${ }^{a}$ George W. Woodruff School of Mechanical Engineering, Georgia Institute of Technology, 771 Ferst Drive, Atlanta, Georgia 30332, USA. E-mail. sgraham@gatech.edu; cola@gatech.edu

${ }^{b}$ California Institute of Technology, 1200 E. California Blvd. MC 309-81, Pasadena, CA 91125, USA

'School of Materials Science and Engineering, Georgia Institute of Technology, 771 Ferst Drive, Atlanta, Georgia 30332, USA

$\uparrow$ Electronic supplementary information (ESI) available: In situ SEM videos of flat-punch nanoindentation into a sample of each CNT forest type, videos of stress-strain curves, and CNT TEM images. See DOI: $10.1039 / \mathrm{c} 2 \mathrm{nr} 30474 \mathrm{k}$ in more detail in order to link their deformation response to their underlying structure.

Some of the existing literature on the mechanical response of CNT forests has investigated the relationship between modulus or stiffness and the morphological variations (i.e., orientation, interaction, and/or density of CNTs) between different forests. ${ }^{8-11}$ None of these studies has considered the synthesisinduced morphological gradients along the height of the forests that can produce gradients in mechanical properties; although the presence of such morphological gradients has been reported. ${ }^{12,15,34,35}$ Some other reports have focused on the deformation of CNT forest pillars ${ }^{10,12-14}$ and continuous films, ${ }^{15-17}$ and these studies revealed coordinated, localized periodic buckling of the CNTs as the dominant deformation mechanism. The local buckling within the forests has been reported to begin at either the bottom (i.e., close to the substrate) $)^{12,14,15,17}$ or top ${ }^{16,17}$ of the CNT forests. While correlations between CNT morphology (e.g., density and orientation) variations along the height and the mechanism of the deformation within CNT forests have been proposed, ${ }^{12,15-17}$ virtually all of them are based on testing either CNT forests produced from one growth condition ${ }^{12,17}$ or a limited number of CNT forests with completely different types of CNTs (e.g., substantially different CNT diameters). ${ }^{16}$ Therefore, mechanical testing of CNT forests that differ only in the vertical morphological gradients while maintaining all other aspects constant is required to investigate the connection between the structural variations within a forest and the mechanical response of the forest.

In this work, we report the results of a series of in situ flatpunch indentation experiments on CNT forests with distinct 
microstructural vertical gradients performed inside a dedicated nano-mechanical instrument, SEMentor, comprised of a scanning electron microscope (SEM) and a nanoindenter module. ${ }^{36}$ The microstructure of CNT forests was varied by changing CNT growth time, using different conditions for CNT growth (e.g., pressure, gas flow rates, etc.), or by using a plasma treatment to create different catalyst morphologies prior to growth. The main differences between the forests studied were the relative change of morphology along the height of forests while the diameter of the CNTs was very similar between all forests. The location of initial instability (i.e., lowest critical buckling load) or bending for CNT forests under a compressive load is revealed through the in situ analysis which allows for detailed comparisons between all samples.

\section{B Experimental section}

Three types of CNT forests were grown on Si substrates coated with Ti $(30 \mathrm{~nm}) / \mathrm{Al}(10 \mathrm{~nm}) / \mathrm{Fe}(3 \mathrm{~nm})$ by electron beam evaporation. Low pressure CVD (LPCVD), near atmospheric pressure CVD (APCVD), and plasma pretreated LPCVD (P-LPCVD) recipes were used for synthesis of $\mathrm{CNT}$ forests in a commercial chemical vapor deposition system (Black Magic Pro 4", Aixtron $\mathrm{SE})$. The growth temperature was $\sim 750{ }^{\circ} \mathrm{C}$ and acetylene was used as the carbon source gas in all three recipes. The chamber pressure was approximately $10 \mathrm{mbar}$ in LPCVD using a $\mathrm{C}_{2} \mathrm{H}_{2}-\mathrm{H}_{2}$ mixture and 720 mbar in APCVD using a $\mathrm{C}_{2} \mathrm{H}_{2}-\mathrm{H}_{2}-\mathrm{N}_{2}$ mixture. The P-LPCVD recipe was a modification of the LPCVD recipe that included a $120 \mathrm{~W}$ hydrogen plasma pretreatment of the catalyst layer for 5 min prior to growth. Each recipe produced multiwall CNTs with diameter ranges determined by a transmission electron microscope (TEM) to be $7.2 \pm$ $1.4 \mathrm{~nm}$ for LPCVD CNTs, $8.8 \pm 2.1 \mathrm{~nm}$ for APCVD CNTs, and $9.3 \pm 5.5 \mathrm{~nm}$ for P-LPCVD CNTs. The average number of walls was measured to be 6, 5, and 5 for LPCVD, APCVD, and P-LPCVD CNT forests, respectively. Aerial densities at the beginning of the growth were expected to be close for LPCVD and APCVD since the catalyst profiles are similar. However, the different growth conditions were used to create two different morphologies along the height of the forests as the pressure influences the growth rate and structural morphology within the forests. This plasma pretreatment acts to remove some catalyst particles and resulted in a forest with lower density. Details of the growth conditions for each sample are listed in Table 1.

Nanoindentation was performed in a custom-built in situ nanomechanical testing instrument, SEMentor, comprised of a nanoindenter (Nanomechanics Inc.) inside a field emission scanning electron microscope (SEM) (FEI Quanta200 FEG). ${ }^{36}$
All samples were loaded and unloaded at a constant displacement rate of $50 \mathrm{~nm} \mathrm{~s}^{-1}$ using a custom-machined, rectangular cross-section $(60 \mu \mathrm{m} \times 80 \mu \mathrm{m})$ diamond flat punch indenter tip. Nanoindentation experiments were performed by loading to a maximum displacement of 20-30 $\mu \mathrm{m}$ followed by complete unloading. The instrument's contribution to the measured raw load-displacement response was obtained by performing an identical test in vacuum and subtracting the data from the CNT forest experimental data. Additional details on the SEMentor and steps ensuring measurement accuracy are presented in ref. 12.

Besides the tests performed in the bulk regions of the CNT films, several indentations were performed along the crosssectional edges for each sample in order to visualize the deformation (see Fig. 1). While the boundary conditions and constraints for these on-edge indentations are different from the in-bulk tests, they are helpful to elucidate some of the specific aspects of deformation mechanisms for each growth condition. A minimum of two on-edge indentations were performed on each sample and all observed deformations for a given sample were similar. Moreover, the distance between the indentation points were large enough (a few times larger than the indenter width of $80 \mu \mathrm{m})$ to prevent adjacent locations on a sample from influencing deformation characteristics. Also, in-bulk locations were selected as far as possible from the edge to ensure that the deformation was not affected by the free edge.

\section{Results and discussion}

LPCVD CNT forests of three heights (70, 190, and $280 \mu \mathrm{m})$ were indented to depths of $20-30 \mu \mathrm{m}$. The axial indentation strain was calculated by dividing the measured vertical displacement by the initial height of each forest. The axial indentation stress was calculated by dividing the applied force by the area of contact between the indenter and the surface of CNT forests. The use of a flat punch makes the calculation of the axial indentation stress more straightforward because the apparent contact area does not change during indentation, as it does for sharp indenter tips like Berkovich or cube corner indenters.

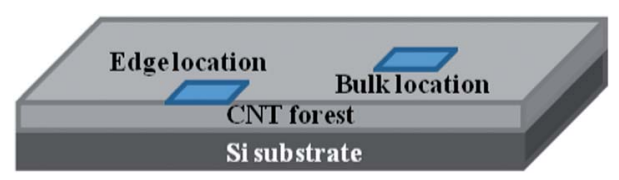

Fig. 1 Schematic diagram (not to scale) of on-edge and in-bulk locations.

Table 1 Parameters for LPCVD, APCVD, and P-LPCVD recipes

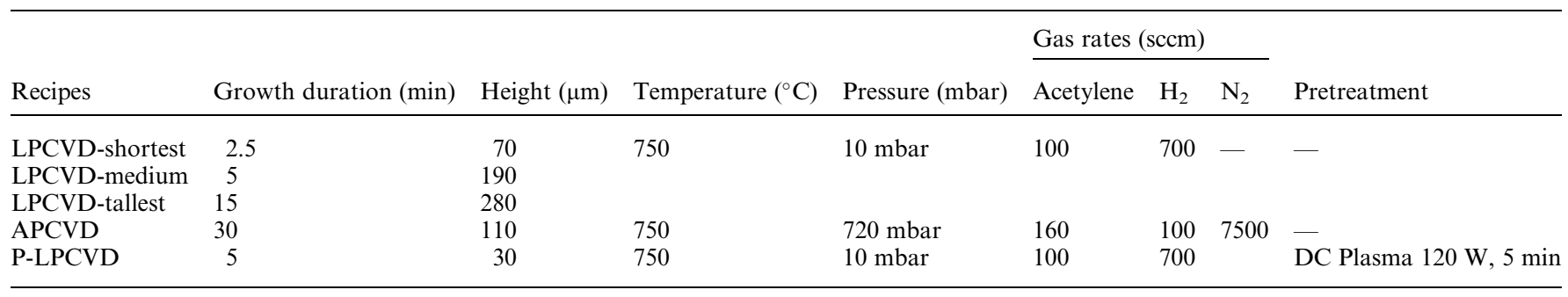


The deformation of the CNT forests after indentation is shown in Fig. 2. In the case of $70 \mu \mathrm{m}$ and $190 \mu \mathrm{m}$ tall LPCVD forests, it appears that the local progressive buckling started at the bottom of the forest at the very beginning of the compression tests. Multiple curved shape buckles formed close to the substrate as shown in Fig. 2a and b. This region for the $190 \mu \mathrm{m}$ forest is magnified in Fig. 2d. The buckles exceeded the projection of the indenter cross-sectional width and the widest buckles are as large as 2-4 times the width of the indenter. The characteristic wavelength of the buckles was about $1-4 \mu \mathrm{m}$, in the same range as previous work with similar CNT types. ${ }^{16,17}$ The bottom progressive buckling continued from the very low measured strain to about $0.10-0.15$ strain. At this point, a small number of buckles formed along the height and vertical shear off-sets started forming along the vertical projection line of the indenter edges. For the case of a $280 \mu \mathrm{m}$ tall LPCVD forest, the position of the first local buckle was not at the bottom, as is the case for the forests with $70 \mu \mathrm{m}$ and $190 \mu \mathrm{m}$ heights, but rather it was approximately $100 \mu \mathrm{m}$ above the bottom of the CNT forest as shown in Fig. 2c. Also, a single buckle formed at the initial buckling location as opposed to formation of multiple buckles close to the substrate (Fig. 2d) in the case for the two shorter forests.

The typical stress-strain curves for the on-edge indentation of LPCVD forests with different heights are shown in Fig. 3a. The initial sections of the stress-strain curves were linear with similar slopes for the samples of all three heights (Fig. 3a) followed by a nonlinear deformation response that depended on the height of the forest. Again all samples were deformed to approximately the same total depth, but the strain is smaller in samples with longer CNTs because the deformation is normalized by the CNT height for calculation of strain. In Fig. 3b, the stress-strain curves for the on-edge and in-bulk locations on a $70 \mu \mathrm{m}$ tall LPCVD forest are compared. As expected, the maximum applied force was

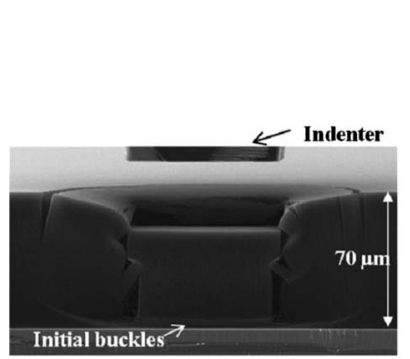

(a)

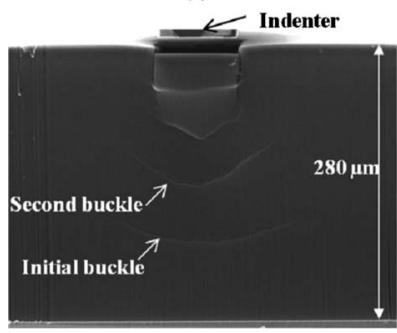

(c)

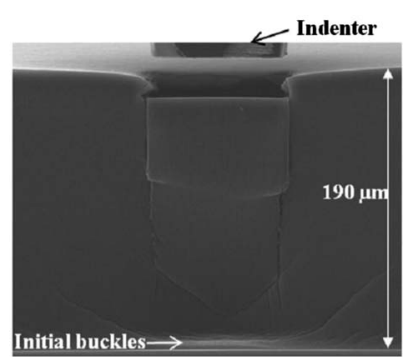

(b)

(d)

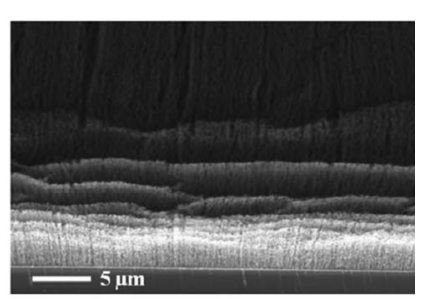

Fig. 2 CNT forest edge after indentation of the LPCVD forest. (a) Shortest, (b) medium, and (c) tallest forest, and (d) magnified view of local buckles close to the substrate in the medium case which is also representative of the shortest case.

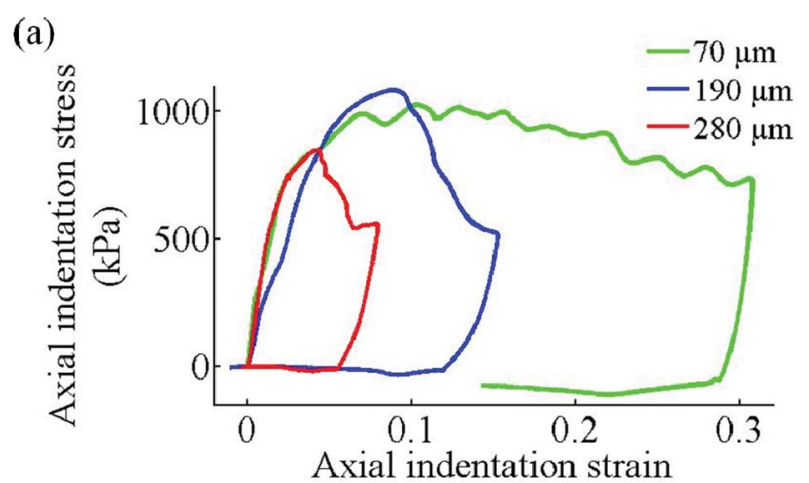

(b)

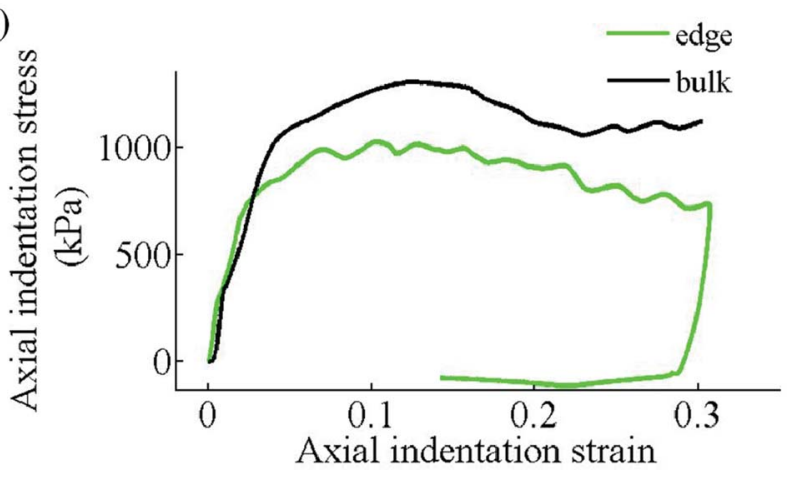

Fig. 3 (a) Axial indentation stress-strain curves for indentation on the edges of LPCVD forests of three heights. (b) Axial indentation stressstrain curves for the shortest LPCVD forest for edge and bulk points.

higher for the bulk locations as compared with the edge due to the boundary constraints in the former. For the $70 \mu \mathrm{m}$ tall forest the maximum stress was $1.3 \mathrm{MPa}$ for a bulk point compared to $1 \mathrm{MPa}$ for a point on the edge as shown in Fig. 3b. Again the slopes of the initial linear sections were similar. Based on our in situ observations, it appears that the points of discrete drops in load likely correspond to the initiation of buckles or vertical shear offsets. Further, we find that similar global softening

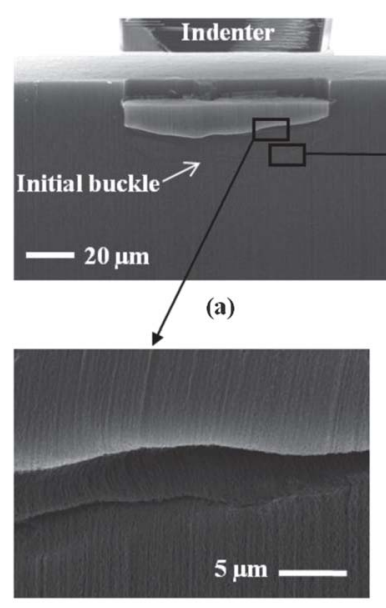

(c)

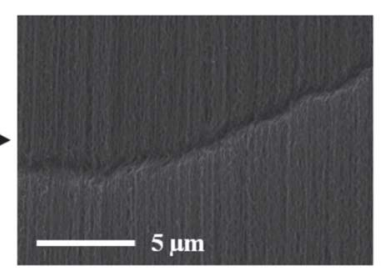

(b)

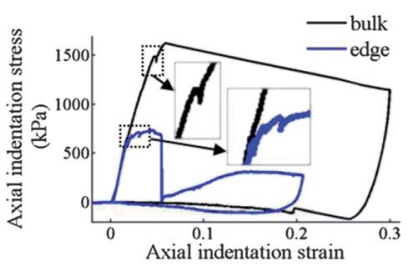

(d)
Fig. 4 (a) APCVD CNT forest edge after indentation, (b and c) high magnification view of the details of buckles inside rectangles in (a), and (d) axial indentation stress-strain curves for edge and bulk points. 
regions after the peak points in the stress-strain curves for 190 and $280 \mu \mathrm{m}$ tall forests were caused by the instabilities due to the formation of additional buckles above the initial one(s), as shown in Fig. 2. The undulations in the curve for the $70 \mu \mathrm{m}$ tall forest were related to the progressive buckling initiated from the substrate. These undulations were very similar to previous observation of undulations in a stress-strain curve due to periodic buckling initiated from the substrate in the case of CNT pillars. ${ }^{12,14,17,37}$ Formation of periodic buckles initiated from the substrate in the case of the medium height $(190 \mu \mathrm{m})$ CNT forest did not cause such prominent undulations in the stress-strain curve as in the case of the short $(70 \mu \mathrm{m})$ height CNT forest. This is likely due to the effect of the larger CNT forest height, which makes the ratio of the buckled region to the entire height smaller and causes a smaller decrease in load via buckling.

A typical deformed APCVD CNT forest is shown in Fig. 4a-c. When a APCVD CNT forest with a height of $110 \mu \mathrm{m}$ was indented on the edge to a strain of $\sim 0.02$, the initial buckle formed at a distance of $40-50 \mu \mathrm{m}$ from the top surface representing $\sim 35$ to $45 \%$ of the total height. Such formation of the first buckle in the upper half of the APCVD forest did not occur in any LPCVD forests, suggesting the existence of significant differences in the forest morphology between these two types of CNT forests. A zoomed-in view of the region where the initial buckle occurred is shown in Fig. 6b. The typical stress-strain curves for locations on the edge and in the bulk of the APCVD forest are shown in Fig. 4d. The stress-strain data from the edge showed a very small drop in stress at a strain of approximately 0.02 as a result of the initiation of the first buckle in the forest. We assume that the similar drop in load in the bulk indentation curve at a strain of about 0.04 was due to the formation of buckles as well.

At a strain of $\sim 0.05$, the second buckle formed $\sim 10 \mu \mathrm{m}$ above the first one in the APCVD samples, upon which the compressed section sheared off vertically underneath the indenter edges. The

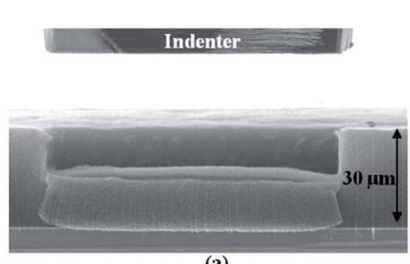

(a)

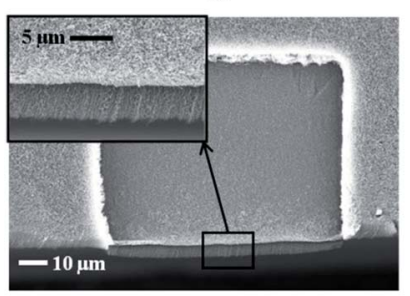

(c)

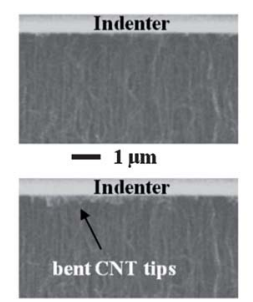

(b)

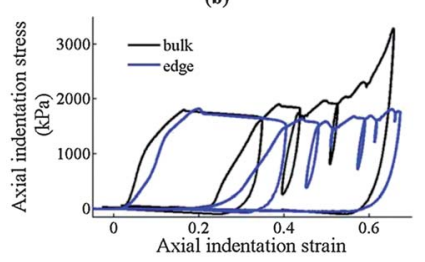

(d)
Fig. 5 Indentation of the P-LPCVD forest on edge. (a) Side view of the deformed spot, (b) side magnified view of the section under indenter before (top image) and after (bottom image) appearance of CNT tips, (c) top view of the deformed spot with magnified view of the out of plane deformation in the drawn rectangle, and (d) axial indentation stressstrain curves for edge and bulk points. larger drops in stress - on the order of $500-800 \mathrm{kPa}-$ for both curves correspond to these points. Fig. $4 \mathrm{c}$ shows a magnified view of the out-of-plane deformation and the location of the second buckle. Notably, the drop in the load for the edge indentation was 1.5 times that in the bulk of the sample, probably as a result of reduced side constraints, which allowed the separated block to deform (see Fig. 4a). The sheared-off block of the CNT forest under the indenter (Fig. 4a) is smaller than that of LPCVD forests. This precipitous drop in load was not observed in the stress-strain curves for LPCVD forests due to the small block of the deformed APCVD sample separating from the parent matrix as opposed to the LPCVD forests, where this did not occur.

SEM images of the deformed P-LPCVD forests and a typical stress-strain curve for the on-edge and in-bulk points are shown in Fig. 5. This deformation started with bending of the CNT tips in contrast to what was seen in the LPCVD samples. The top image in Fig. 5b is the indenter in contact with CNT tips at the beginning of deformation and the bottom image shows the same point after additional displacement of the indenter causes CNT tips to bend enough to appear clearly in the image. At the strain of $\sim 0.15$ to 0.2 , two buckles formed - one in the top $20 \%$ and another - in the bottom $80 \%$ of the forest height for both edge and in-bulk curves. The formation of these buckles corresponds to the first plateau region followed by the first hysteretic loop in the curves shown in Fig. 5d.

The out-of-plane deformation that was increased by continued displacement after formation of buckles is shown in the top-view SEM images in Fig. 5c. We attribute the formation of additional hysteretic loops in the stress-strain curves with further compression of the CNT forest to buckling of segments of the forest under the indenter that could not be visualized in our testing (these segments are covered from view by the buckled layer). The initial parts of the curves for the indentation test of the P-LPCVD forest both on the edge and in-bulk are similar, as is the case for the APCVD and shortest LPCVD forest; however, the extra constraints on out-of-plane displacement in the in-bulk testing lead to higher stiffness at larger displacements as shown in Fig. 5d.

The change of the growth recipes resulted in significantly different morphologies along the height of the CNT forests. The aforementioned deformation mechanisms of the CNT forests are connected to the different microstructures along their crosssectional height. The change of microstructure of the LPCVD forests with height is demonstrated in the high magnification SEM images in Fig. 6. The images are taken from three locations near the top, bottom, and a point in between which is at the middle of the 70 and $180 \mu \mathrm{m}$ tall CNT forests and below the middle where the first buckle formed in the $290 \mu \mathrm{m}$ CNT forest. The images illustrate well-aligned and high density CNTs on the top sections of the CNT forests with three different heights. CNTs appear to become more tortuous at the middle and bottom sections of the forests. CNT aerial density has been reported in the literature ${ }^{34}$ to decrease from top to the bottom of the CNT forests. The reason for the loss of adhesion between some CNTs and the substrate during growth, causing the aerial density decay, is yet to be investigated. The lower aerial density at the bottom part is likely the reason for formation of the first buckles close to the substrate in the 70 and $180 \mu \mathrm{m}$ tall CNT forests. Low density has also been suggested as the dominant factor in previous 

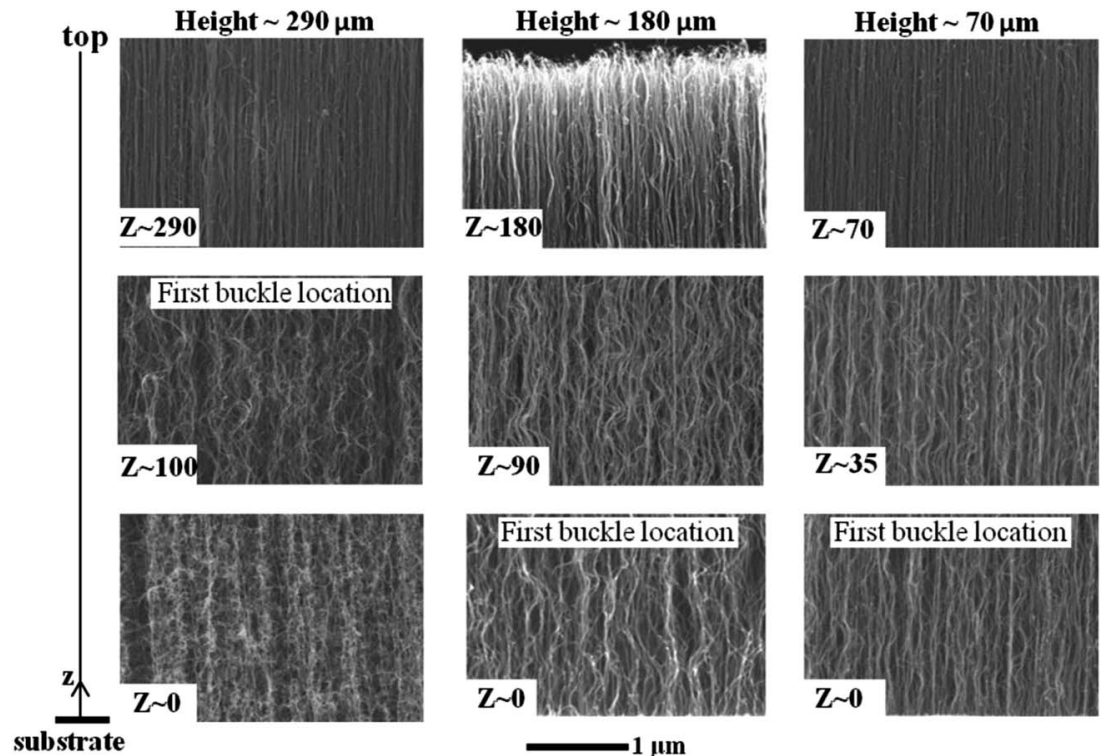
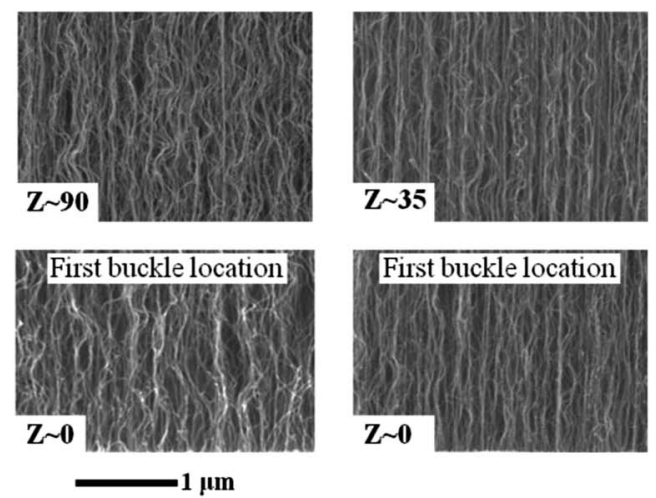

Fig. 6 SEM images at $80 \mathrm{k} \times$ magnification showing orientation and entanglement of CNTs along the height of LPCVD CNT forests with three different heights - left: $290 \mu \mathrm{m}$, middle: $180 \mu \mathrm{m}$, and right: $70 \mu \mathrm{m}$. The scale bar is $1 \mu \mathrm{m}$ for each image. The distance from the substrate $(Z)$ is labeled in each image. The locations of the first buckle are also marked.

studies of the formation of buckles close to the substrate. ${ }^{12,15,17}$ Interestingly, the buckles formed approximately $100 \mu \mathrm{m}$ above the substrate in the $290 \mu \mathrm{m}$ tall CNT forest. The SEM images depicted in Fig. 6 show a visually discernible unique morphology close to the substrate of the $290 \mu \mathrm{m}$ CNT forest. High tortuosity of the CNTs close to the substrate is the morphological characteristic of a catalytic CVD grown CNT forest that is self-terminated or approaching the self-termination point. ${ }^{34,35}$ The lower average growth rate of this forest compared to the shorter forests is also an indication of approaching self-termination. ${ }^{35} \mathrm{We}$ hypothesize that the reason for the formation of the first buckle $\sim 100 \mu \mathrm{m}$ above the substrate is the existence of this tangled layer. The tortuosity of the CNTs decreases by distance from the substrate. The CNTs about $100 \mu \mathrm{m}$ above the substrate are less tortuous and appear to be more similar in directionality to those observed in the locations of first buckles in the 70 and $180 \mu \mathrm{m}$ tall CNT forests.

Three locations along the cross-sectional height of CNT forests grown with APCVD and P-LPCVD recipes - close to the top, middle, and bottom - were imaged using an SEM. In Fig. 7, these SEM images are compared with those taken from a $70 \mu \mathrm{m}$ tall LPCVD forest. The height of the APCVD CNT forest (110 $\mu \mathrm{m})$ lies between the heights of the two shorter tested LPCVD CNT forests $(70,180 \mu \mathrm{m})$. However, the first buckle did not initiate from the bottom as it did for the two LPCVD CNT forests. It formed slightly above the middle of the height approximately $40-50 \mu \mathrm{m}$ from the top surface. The location of the first buckle, as shown in Fig. 7, appears to have an average orientation similar to that of the location at the middle and bottom of the $70 \mu \mathrm{m}$ tall LPCVD forest. In the APCVD forest, the CNTs tortuosity increases from the location of the first buckle to the bottom of the forest. The high degree of tortuosity of the CNTs at the bottom half of the height is the possible reason for the first buckle to not form at the bottom of the CNT forest despite the likely lower CNT number density in that location. The increase in tortuosity is likely due to approaching self-termination in the growth of the APCVD forest. ${ }^{34,35}$

The bending of tips, formation of a local buckle near the top of the forest, and formation of a large buckle under the top buckle in the P-LPCVD forest did not occur in the LPCVD and APCVD CNT forests. We attribute the bending of tips and the formation of a local buckle near the top of this CNT forest to the much higher degree of alignment of CNT tips compared to that of the middle and bottom sections of this CNT forest. This significant change of alignment of CNTs from the top to the middle of the P-LPCVD CNT forest was not observed in the other two CNT forest types. A high degree of uniform tortuosity of the CNTs in the middle and bottom sections of the P-LPCVD forest is the likely cause of the formation of the large buckle that follows under the top buckle. This did not occur in the other two CNT forest types presumably because of the larger gradients along their heights. The unique microstructure of the P-LPCVD forest is probably due to the effect of plasma in removing some catalyst particles and creating a CNT forest that appears to have a smaller aerial density than a LPCVD CNT forest. P-LPCVD CNTs were shorter than the other two types (LPCVD and APCVD) due to an early self-termination. High tortuosity of CNTs might be the cause of early self-termination. ${ }^{35}$ The deformation mechanism appears to be dependent upon the unique microstructure of CNTs rather than the shorter height. CNT forests with similar morphology (aligned CNTs on top of highly tortuous CNTs) and different heights are expected to deform in a similar manner.

The above observations suggest that highly tortuous CNTs in any location along the height of any of the CNT forests (e.g. at the bottom of the APCVD and $290 \mu \mathrm{m}$ tall LPCVD CNT forests, and at the middle and bottom of the P-LPCVD forest) were not prone to buckling. This is possibly due to the extra constraints 

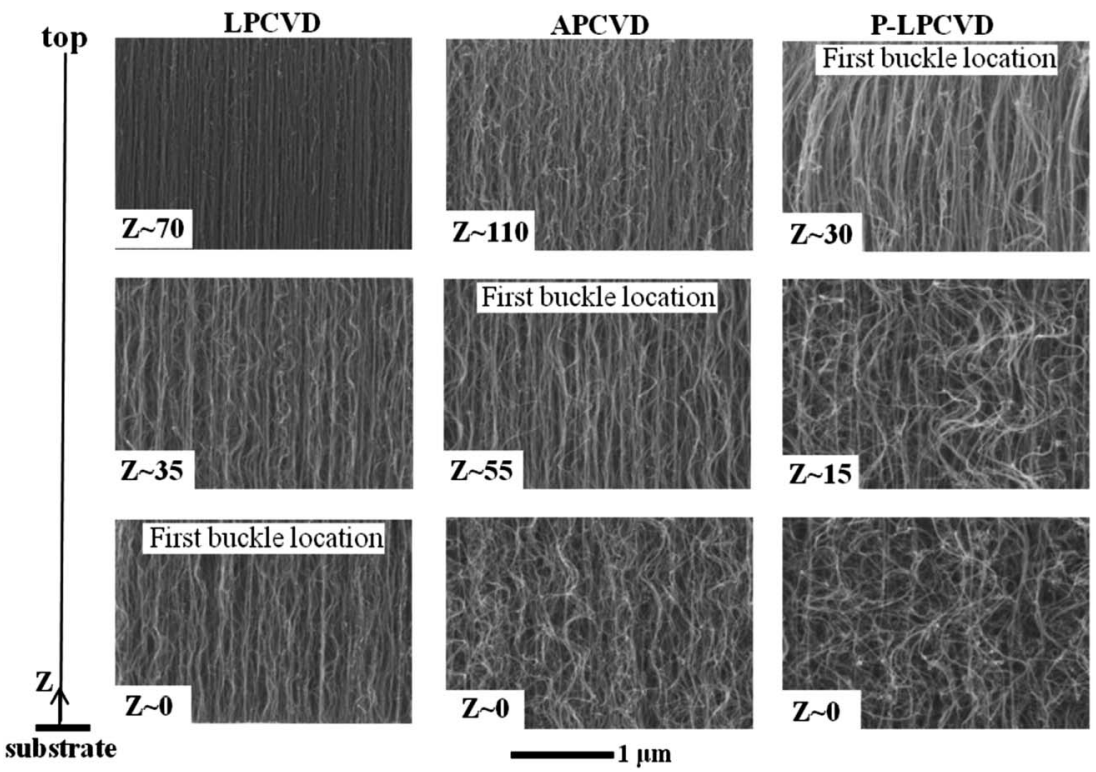

Fig. 7 SEM images at $80 \mathrm{k} \times$ magnification showing orientation and entanglement of CNTs along the height of LPCVD, APCVD, and P-LPCVD CNT forests. The distance from the substrate $(Z)$ is labeled in each image and the images from the locations of the first buckle are also marked.

that result from the greater number of contacts between the adjacent CNTs. Less interaction between the CNTs in the less tortuous sections caused the CNTs to be more prone to bending and buckling. The CNT forests without highly tortuous CNTs (e.g. 70 and $180 \mu \mathrm{m}$ tall LPCVD CNT forests), however, buckled at the bottom sections, which are the locations of lowest CNT aerial density. Thus, we conclude that aerial density and tortuosity are two factors dictating the location of the initial instability in the CNT forests. The first buckle forms close to the base of the CNTs, which is the location of the lowest aerial density, unless the CNTs are highly tortuous.

Raman spectroscopy of the samples demonstrates a decay of the G/D ratio from the top to bottom of the CNT forests as it has also been reported in previous works. ${ }^{34}$ This indicates a decrease in the quality of CNTs likely due to the increase in defect density due to higher tortuosity. The defect density, however, is not correlated to the location of the initial buckles. The highly tortuous CNTs are less prone to buckling despite their likely higher defect density.

In addition to the differences in the mechanism of deformation of CNT forests, there are differences in other mechanical parameters for each forest type as summarized in Table 2 . Stiffness is one of the parameters. Table 2 shows that the stiffness of CNT forests is influenced primarily by the height of the forests. With the maximum displacement fixed at 20-30 $\mu \mathrm{m}$ for all compression tests, the shorter CNT forests were deformed to higher strains, and hence - closer to the incompressible height of the forest ${ }^{38}$ which gave the forest a stiffer overall response. The unloading portions of load-displacement curves correspond to the elastic recovery. Therefore, the slopes of the initial portions of unloading of the in-bulk locations were used to calculate the unloading stiffnesses and the effective elastic moduli of the different CNT forest types, as commonly done in nanoindentation studies. ${ }^{39,40} \mathrm{We}$ find that the elastic modulus of 90-120 MPa is virtually independent of forest height, consistent with previous observations. ${ }^{19}$ Calculation of modulus using stiffness and indenter area with the assumption of indentation of an elastic half-space (as is commonly done in nanoindentation experiments on monolithic materials) is not suitable for this type of indentation that involves separation of a block of CNTs from the rest of the forest. The importance of studying the deformation mechanisms of forests and not relying strictly on their elastic modulus for use in mechanical applications is emphasized by the fact that elastic modulus is practically constant, and independent of morphology and height for the three different types of CNT forests studied here.

Other significant differences among the samples tested here were the amount of elastic recovery of the deformation,

Table 2 Unloading stiffness, effective elastic modulus, elastic recovery ratio, largest buckle width ratio, and maximum value of negative stress during unloading for different CNT forests

\begin{tabular}{lcrrr}
\hline Recipe & LPCVD-shortest & LPCVD-tallest & APCVD & P-LPCVD \\
\hline Average height $(\mu \mathrm{m})$ & 70 & 285 & 110 & 30 \\
Unloading stiffness $\left(\mathrm{N} \mathrm{m}^{-1}\right)$ & 6240 & 1680 & 4560 & 105 \\
Effective elastic modulus $(\mathrm{MPa})$ & 91 & 100 & 200 \\
Elastic recovery ratio $(\%)$ & 13 & 55 & 46 & 120 \\
Largest buckle width ratio & 2 & 3 & 45 & 1 \\
Maximum negative stress $(\mathrm{kPa})$ & 53 & 22 & 166 \\
\hline
\end{tabular}


magnitude of the "negative stress" during the unloading steps, and the extent to which CNTs not directly under the indenter deformed. These characteristics are quantified in Table 2. Elastic recovery ratio was calculated by dividing the magnitude of recovered displacement by the maximum displacement using data from tests on the sample edge. The magnitude of recovered displacement was measured using recorded videos of indentation of CNT forests (see the ESI $\dagger$ ) by subtracting the permanent displacement of the CNT forest sample from the maximum indentation depth. The elastic recovery was lowest $(13 \%)$ for the shortest LPCVD forest and highest (55\%) for the tallest LPCVD forest. If the formed buckles remained permanent, the lowest recovery for the shortest LPCVD may be explained by the presence of the largest number of buckles in the deformed regions. This is consistent with only a few buckles having been formed in the tallest LPCVD forest and both the APCVD and P-LPCVD forests, all of which have similar recovery ratios $(45-55 \%)$. The ratio of the width of the largest buckle, measured for the on-edge indentation points, to the width of the indenter shows the extent of deformation to CNTs not directly under the indenter. This parameter was close to 1 for P-LPCVD and APCVD forests, which means only an area equal to the area of the indenter was deformed. The buckle width to indenter width ratio was the highest for the LPCVD forests. This difference may be because of the location of first buckles farther from the indenter for the LPCVD forest and also because of the relatively high CNT entanglement at the top surface of the LPCVD forest compared to the other two types. The apparent negative stress arising from negative measured loads during the final stages of unloading is likely a result of adhesion between the diamond indenter tip and the tops of the CNT forest, as commonly observed previously. ${ }^{9,20,24}$ The maximum negative stress was measured for in-bulk points and is much higher for the APCVD forest than the other two types. Additional investigations are warranted to clearly explain the effects of CNT forest morphology (global and local) on the elastic recovery ratio, negative stress, and extent of deformation of CNTs not under the indenter.

\section{Conclusions}

In summary, we synthesized CNT forests with three different heights and also three types of CNT forests to elicit samples with distinct morphologies, all of which were subjected to identical in situ flat punch nanoindentation. We find that CNT aerial density and tortuosity represent two key factors governing the overall mechanical response of the CNT forests to local compressive loading. We find that all CNT forests undergo an initial instability manifested as the formation of a buckle. These local buckles that determine a major part of the deformation mechanism of CNT forests form at the location of the best combination of low density and less tortuous CNTs along the height. We show that the location of initial buckling moves up from the bottom of the forest if the CNTs near the substrate are highly tortuous. The LPCVD forests begin to buckle at the lower half of the forest while the first buckle in the APCVD is formed close to the middle. P-LPCVD forests buckled in yet another way, with the formation of a smaller buckle at the top and a larger one beneath it. We believe that this study provides insights into establishing a link between the differences in the density and tortuosity along the CNT forest height and the distinct deformation mechanisms, which suggest that new synthesis methods could be developed to control the CNT forest morphology and tailor the mechanical response to suit the needs of specific applications.

\section{Acknowledgements}

This work was supported by seed funding from the Georgia Institute of Technology. The authors gratefully acknowledge the research assistance of John $\mathrm{H}$. Taphouse and experimental support from Dr Jason Nadler. JRG gratefully acknowledges the financial support of the Army Research Office through the Institute for Collaborative Biotechnologies and Sid Pathak for helpful discussions.

\section{References}

1 W. A. de Heer, A. Châtelain and D. Ugarte, Science, 1995, 270, 1179. 2 J. Xu and T. S. Fisher, Int. J. Heat Mass Transfer, 2006, 49, 1658.

3 P. B. Amama, B. A. Cola, T. D. Sands, X. Xu and T. S. Fisher, Nanotechnology, 2007, 18, 385303.

4 M. Park, B. A. Cola, T. Siegmund, J. Xu, M. R. Maschmann, T. S. Fisher and H. Kim, Nanotechnology, 2006, 17, 2294.

5 Z. P. Yang, L. Ci, A. James, S. Y. Lin and P. M. Ajayan, Nano Lett., $2008,8,446$.

6 X. Wang, L. Wang, O. Adewuyi, B. Cola and Z. Zhang, Appl. Phys. Lett., 2010, 97, 163116.

7 T. Xu, Z. Wang, J. Miao, X. Chen and C. M. Tan, Appl. Phys. Lett., 2007, 91, 042108

8 H. Malik, K. J. Stephenson, D. F. Bahr and D. P. Field, J. Mater. Sci., 2011, 46, 3119.

9 C. M. McCarter, R. F. Richards, S. D. Mesarovic, C. D. Richards, D. F. Bahr, D. McClain and J. Jiao, J. Mater. Sci., 2006, 41, 7872.

10 A. Qiu, D. F. Bahr, A. A. Zbib, A. Bellou, S. D. Mesarovic, D. McClain, W. Hudson, J. Jiao, D. Kiener and M. J. Cordill, Carbon, 2011, 49, 1430.

11 J. R. Raney, A. Misra and C. Daraio, Carbon, 2011, 49, 3631.

12 S. B. Hutchens, L. J. Hall and J. R. Greer, Adv. Funct. Mater., 2010, 20, 2338 .

13 O. Yaglioglu, PhD thesis, Massachusetts Institute of Technology, 2007.

14 A. A. Zbib, S. D. Mesarovic, E. T. Lilleodden, D. McClain, J. Jiao and D. F. Bahr, Nanotechnology, 2008, 19, 175704.

15 A. Y. Cao, P. L. Dickrell, W. G. Sawyer, M. N. Ghasemi-Nejhad and P. M. Ajayan, Science, 2005, 310, 1307.

16 M. R. Maschmann, Q. Zhang, R. Wheeler, F. Du, L. Dai and J. Baur, ACS Appl. Mater. Interfaces, 2011, 3, 648.

17 M. R. Maschmann, Q. H. Zhang, F. Du, L. M. Dai and J. Baur, Carbon, 2011, 49, 386.

18 H. J. Qi, K. B. K. Teo, K. K. S. Lau, M. C. Boyce, W. I. Milne, J. Robertson and K. K. Gleason, J. Mech. Phys. Solids, 2003, 51, 2213.

19 T. Tong, Y. Zhao, L. Delzeit, A. Kashani, M. Meyyappan and A. Majumdar, Nano Lett., 2008, 8, 511.

20 S. D. Mesarovic, C. M. McCarter, D. F. Bahr, H. Radhakrishnan, R. F. Richards, C. D. Richards, D. McClain and J. Jiao, Scr. Mater., 2007, 56, 157.

21 H. Radhakrishnan, S. Mesarovi, C. Mccarter, D. Bahr and C. Richards, Metalurgija, 2007, 13, 301.

22 C. Cao, A. Reiner, C. Chung, S. H. Chang, I. Kao, R. V. Kukta and C. S. Korach, Carbon, 2011, 49, 3190.

23 H. Qi, K. Teo, K. Lau, M. Boyce, W. Milne, J. Robertson and K. Gleason, J. Mech. Phys. Solids, 2003, 51, 2213.

24 A. Qiu, S. Fowler, J. Jiao, D. Kiener and D. Bahr, Nanotechnology, 2011, 22, 295702.

25 J. Suhr, P. Victor, L. Ci, S. Sreekala, X. Zhang, O. Nalamasu and P. Ajayan, Nat. Nanotechnol., 2007, 2, 417.

26 A. Zbib, S. D. Mesarovic, E. Lilleodden, D. McClain, J. Jiao and D. Bahr, Nanotechnology, 2008, 19, 175704. 
27 S. Pathak, Z. G. Cambaz, S. R. Kalidindi, J. G. Swadener and Y. Gogotsi, Carbon, 2009, 47, 1969.

28 Q. Zhang, Y. Lu, F. Du, L. Dai, J. Baur and D. Foster, J. Phys. D. Appl. Phys., 2010, 43, 315401.

29 R. L. D. Whitby, S. V. Mikhalovsky and V. M. Gun'ko, Carbon, 2010, 48, 145.

30 J. Wang and M. Gutierrez, Micro Nano Lett., 2007, 2, 111.

31 C. Daraio, V. F. Nesterenko, S. Jin, W. Wang and A. M. Rao, J. Appl. Phys., 2006, 100, 064309.

32 S. Pathak, E. J. Lim, P. P. S. S. Abadi, S. Graham, B. A. Cola and J. R. Greer, ACS Nano, 2012, 6, 2189.

33 S. B. Hutchens, E. Decolvenaere, S. Pathak and J. R. Greer, Mater. Today, 2012, 15, 127.
34 M. Bedewy, E. R. Meshot, H. C. Guo, E. A. Verploegen, W. Lu and A. J. Hart, J. Phys. Chem. C, 2009, 113, 20576.

35 J. Wu, Y. Ma, Y. Huang, Z. Liu, X. Yang and Y. Chen, Colloids Surf., $A, 2008, \mathbf{3 1 3}, 13$.

36 J. Y. Kim, D. Jang and J. R. Greer, Scr. Mater., 2009, 61, 300.

37 S. B. Hutchens, A. Needleman and J. R. Greer, J. Mech. Phys. Solids, 2011, 59, 2227.

38 B. A. Cola, J. Xu and T. S. Fisher, Int. J. Heat Mass Transfer, 2009, 52, 3490

39 M. Doerner and W. Nix, J. Mater. Res., 1986, 1, 601.

40 W. C. Oliver and G. M. Pharr, J. Mater. Res., 1992, 7, 1564. 